\title{
From ice to dust
}

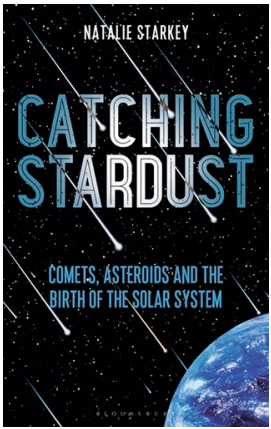

Catching Stardust: Comets, Asteroids and the Birth of the Solar System

By Natalie Starkey

BLOOMSBURY SIGMA: 2018. 256PP. E16.99 a complex place with material travelling both inwards towards and outwards away from the Sun. The second mission described in detail is the European Space Agency's Rosetta mission, which entered orbit around comet 67P/

Churyumov-Gerasimenko (pictured) in 2014. The most dangerous and dramatic part of this mission was when Rosetta's sidekick lander, Philae, attempted the first landing onto the unstable surface of a comet. After bouncing around the irregular surface, the lander came to a stop and survived long enough to beam back several precious packets of data. The data showed that the comet contains a menagerie of molecules including carbon dioxide, carbon monoxide, and numerous nitrogen-bearing and organic species.

As well as being storytellers of our origins, comets and asteroids may provide more practical benefits. They could be exploited for resources that are quickly becoming scarce on Earth, such as metals. They may even provide water for use by missions as part of a multi-stage exploration of the Solar System. A chapter on asteroid mining describes these possibilities, and is a testament to how quickly the technology for extraterrestrial resource exploitation is progressing. Only a few years ago the topic would have been the stuff of science fiction, and now it is material for a non-fiction book.

The final chapter turns to asteroids and comets as bad guys. What could we do to save the Earth if one was careering towards us? It turns out there are several potential options, none of which involves dispatching Bruce Willis to blow it up (robotic missions are likely to be more suitable). All the options sound remarkably improbable to an outsider's eye, yet are being actively researched and may indeed be feasible. Broadly, the space rock might be diverted, or it might be destroyed. Spray-painting it or covering it in foil might divert it by changing the way it interacts with solar rays. Laser-blasting would gently push it into a different orbit. Destroying it could necessitate hitting it with a high velocity impactor or a nuclear weapon, a potentially risky option if there is danger of fragments then raining down on Earth.

There are several popular books about meteorites, and about the asteroids that are parents to most meteorites. Starkey's book is unusual in also considering comets and their role in understanding our origins. It points out that there is plenty of cometary material, in the form of tiny grains, that falls gently onto the surface of the Earth each year. Some of it is collected in high-altitude

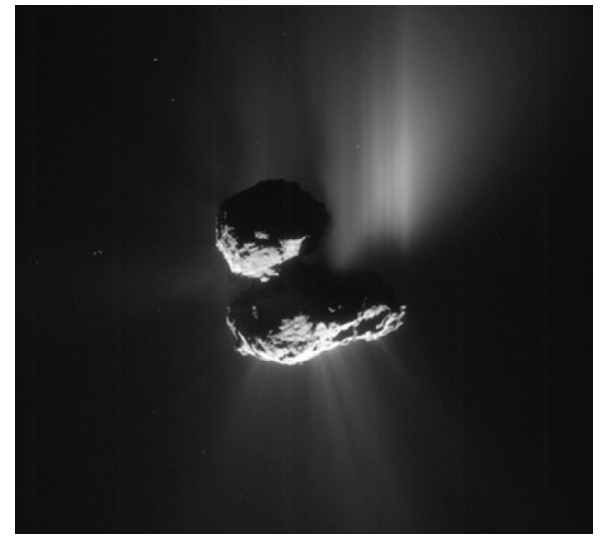

Credit: reproduced from M. Pajola et al. Nat. Astron. 1, 0092 (2017); Macmillan Publishers Ltd.

aeroplanes for us to study. Such material lacks the drama of a meteorite fall and so we are less aware of this material's constant contributions to increasing the Earth's mass.

This book does not read as a textbook. It has a quirky, nonlinear style, and presents information in a very readable, conversational way. It is full of analogies, such as the Solar System as a city containing urbanite inner planets and commuting comets from the suburbs, an analogy that the writer draws on a few times in the book. Starkey's style makes for an easy and charming read. I would recommend it to new graduate students, and to anyone interested in these amazing rocks that bear witness to the history of the Solar System and that bring both new life and epic destruction.

This year is a great one for those of us interested in space rocks and what they can tell us. Two missions are currently on course towards asteroids. NASA's OSIRIS-REx is heading towards asteroid Bennu and its sister mission, Japanese Space Agency's Hayabusa2, is on course for asteroid Ryugu. By the end of 2018 both missions will be at their targets. Both will then sample the asteroidal regolith and return it to Earth for further study; Hayabusa2 is due to return samples in 2020 and OSIRISREx in 2023. These precious samples will help us learn about our origins and give insights into how we may protect our future.

Reviewed by Sara S. Russell

Department of Earth Sciences, Natural History

Museum, London, UK.

e-mail:sara.russell@nhm.ac.uk

Published online: 1 June 2018

https://doi.org/10.1038/s41550-018-0487-z 